\title{
Shortcut-to-adiabaticity quantum Otto refrigerator
}

\author{
Obinna Abah $\odot,{ }^{1}$ Mauro Paternostro, ${ }^{1}$ and Eric Lutz ${ }^{2}$ \\ ${ }^{1}$ Centre for Theoretical Atomic, Molecular, and Optical Physics, School of Mathematics and Physics, \\ Queen's University Belfast, BT7 1NN, United Kingdom \\ ${ }^{2}$ Institute for Theoretical Physics I, University of Stuttgart, D-70550 Stuttgart, Germany
}

(Received 3 February 2020; accepted 6 April 2020; published 4 May 2020)

\begin{abstract}
We investigate the performance of a quantum Otto refrigerator operating in finite time and exploiting local counterdiabatic techniques. We evaluate its coefficient of performance and cooling power when the working medium consists of a quantum harmonic oscillator with a time-dependent frequency. We find that the quantum refrigerator outperforms its conventional counterpart, except for very short cycle times, even when the driving cost of the local counterdiabatic driving is included. We moreover derive upper bounds on the performance of the thermal machine based on quantum speed limits and show that they are tighter than the second law of thermodynamics.
\end{abstract}

DOI: 10.1103/PhysRevResearch.2.023120

\section{INTRODUCTION}

Heat engines and refrigerators are two prime examples of thermal machines. While heat engines produce work by transferring heat from a hot to a cold reservoir, refrigerators consume work to extract heat from a cold to a hot reservoir $[1,2]$. Refrigerators thus appear as heat engines functioning in reverse. According to the second law of thermodynamics, the coefficient of performance (COP) of any refrigerator, defined as the ratio of heat input and work input, is limited by the Carnot expression, $\epsilon_{\mathrm{C}}=T_{1} /\left(T_{2}-T_{1}\right)$, where $T_{1,2}$ denote the respective temperatures of the cold and the hot reservoirs $[1,2]$. However, this maximum coefficient of performance is only attainable in the limit of infinitely long refrigerator cycles when the cooling power vanishes. At the same time, any refrigerator cycle that runs at finite speed necessarily dissipates energy, which leads to irreversible entropy production and a reduction of its coefficient of performance. An important issue is to optimize the finite-time performance of thermal machines [3]. Although the optimal performance of real heat engines is usually characterized when conditions of maximum output power are achieved, there is no clear corresponding criterion for refrigerators [4]. A meaningful figure of merit to consider when aiming to characterize a refrigerator is the optimized product of the coefficient of performance $\epsilon$ and the cooling power of the refrigerator, the coefficient of performance at maximum figure of merit, $\epsilon^{*}=1 / \sqrt{1-T_{1} / T_{2}}-1$ [5-8].

Techniques based on shortcuts to adiabaticity (STA) $[9,10]$ have recently been suggested as promising candidates to approach such a desired regime of performance-optimized finite-time quantum thermal machines. The implementation

Published by the American Physical Society under the terms of the Creative Commons Attribution 4.0 International license. Further distribution of this work must maintain attribution to the author(s) and the published article's title, journal citation, and DOI. of STA methods on an evolving system mimics its adiabatic dynamics in a finite time [10-24]. Among the STA techniques put forward so far is the local counterdiabatic (LCD) driving, which cancels the possible nonadiabatic transitions induced by the dynamics of a given system by introducing an auxiliary local control potential [15]. It offers a wide range of applicability and has been experimentally realized in stateof-the-art ion trap setups [19,20]. Such STA strategies hold the potential to enhance the performance of both classical and quantum heat engines [25-31]. However, these studies have so far mainly focused on the unattainability of zero temperature (according to the third law of thermodynamics) in a quantum refrigerator context [32]. We here intend to perform a full-fledged investigation of the application of STA schemes to enhance the overall performance of a quantum refrigerator. Moreover, the implementation of STA protocols is not without an energetic cost, which is induced by the additional control potentials. In this regard, the cost of performing STA drivings has only recently been included in the performance analysis of quantum heat engines [30,31,33-37].

In this paper, we study the STA quantum Otto refrigerator taking into account the cost of the driving in the performance analysis. We explicitly evaluate the coefficient of performance and the cooling power of such refrigerator. We find that its performance can exceed its conventional counterpart even when the cost of the STA driving is included, except for very short cycle times. We further use the concept of quantum speed limits for driven unitary dynamics [38] to derive generic upper bounds on both the coefficient of performance and the cooling rate of the superadiabatic refrigerator.

The remainder of this paper is organized as follows. In Sec. II we introduce the quantum Otto refrigerator and illustrate the formalism and notation in use in the rest of the paper. Section III is dedicated to the analysis of the quantum refrigerator under local counterdiabatic STA driving with Sec. IV discussing its performance. Section $\mathrm{V}$ is further dedicated to the establishment of upper bounds on such a performance of the refrigerator as set by the use of the quantum speed limit 


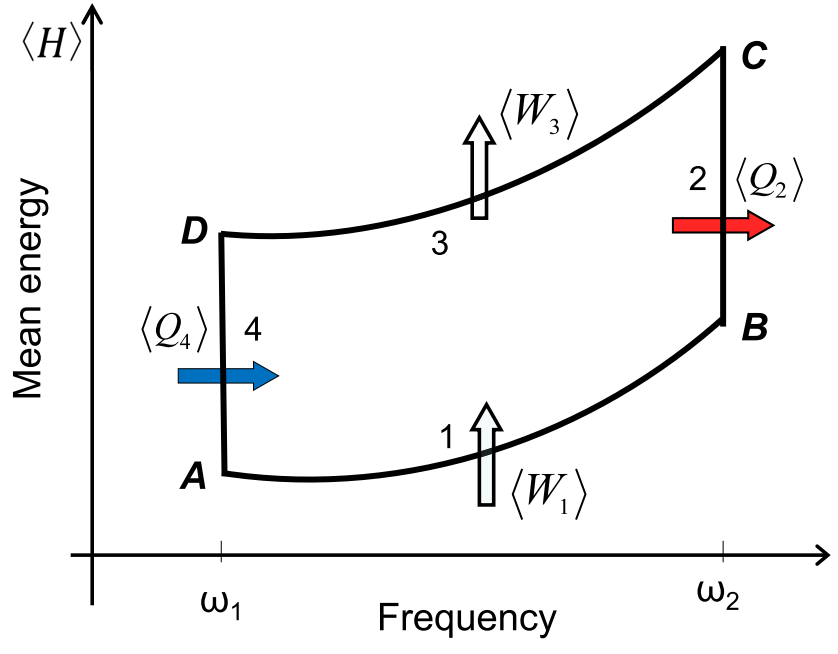

FIG. 1. Energy-frequency diagram of a generic quantum Otto refrigerator. The thermodynamic cycle consists of two adiabatic processes (strokes 1 and 3) and two isochoric processes (strokes 2 and 3). During one complete cycle, work $\left\langle W_{1}\right\rangle+\left\langle W_{3}\right\rangle$ is consumed by the quantum refrigerator to pump heat $\left\langle Q_{4}\right\rangle$ from the cold to the hot reservoir.

valid for the dynamics that we explore here. Finally, in Sec. VI we draw our conclusions and discuss the possibility for further developments opened by our assessment.

\section{QUANTUM OTTO REFRIGERATOR}

The quantum Otto cycle is a paradigm for thermodynamic quantum devices [7,8,27,32,39-48]. The cycle consists of two adiabatic and two isochoric processes. At the end of a cycle, work is consumed by the refrigerator to pump heat from a cold to a hot reservoir. In this paper we make the choice of a working medium embodied by a quantum harmonic oscillator with controllable time-dependent frequency $\omega_{t}$ and corresponding Hamiltonian

$$
H_{\mathrm{O}}(t)=\frac{1}{2 m} p^{2}+\frac{1}{2} m \omega_{t}^{2} x^{2} .
$$

Here $m$ is the mass of the oscillator while $x(p)$ is its position (momentum) operator. The device is alternately coupled to two heat baths at inverse temperatures $\beta_{i}=1 /\left(k_{\mathrm{B}} T_{i}\right)(i=$ $1,2)$, where $k_{\mathrm{B}}$ is the Boltzmann constant. Concretely, the Otto cycle consists of the following four steps as shown in Fig. 1.

(1) Adiabatic compression. Corresponding to the transformation $\mathrm{A}\left(\omega_{1}, \beta_{1}\right) \rightarrow B\left(\omega_{2}, \beta_{1}\right)$ in Fig. 1 . The frequency is varied during time $\tau_{1}$, while the system is isolated from the baths. The corresponding evolution is unitary and the von Neumann entropy of the oscillator is constant.

(2) Hot isochore. Associated with the transformation $\mathrm{B}\left(\omega_{2}, \beta_{1}\right) \rightarrow C\left(\omega_{2}, \beta_{2}\right)$ in Fig. 1 . In this process, the oscillator is weakly coupled to the reservoir at inverse temperature $\beta_{2}$ at fixed frequency and for a time $\tau_{2}$. Notice that no request is made for thermalization of the oscillator.

(3) Adiabatic expansion. Described by the transformation $C\left(\omega_{2}, \beta_{2}\right) \rightarrow D\left(\omega_{1}, \beta_{2}\right)$ in Fig. 1 . The frequency of the work- ing medium is unitarily changed back to its initial value during time $\tau_{3}$. No change of entropy occurs during this stroke.

(4) Cold isochore. At constant frequency, illustrated by the $D\left(\omega_{1}, \beta_{2}\right) \rightarrow A\left(\omega_{1}, \beta_{1}\right)$ process in Fig. 1 . This transformation is obtained by weakly coupling the oscillator to the reservoir at inverse temperature $\beta_{1}>\beta_{2}$ and letting the relaxation to the initial thermal state $A\left(\omega_{1}, \beta_{1}\right)$ occur within a (in general short) time $\tau_{4}$

The total cycle time is $\tau_{\text {cycle }}=\sum_{j=1}^{4} \tau_{j}$. In the rest of our analysis, we assume, as commonly done $[8,32,43,46]$, that the time needed to accomplish the isochoric transformations is negligible with respect to the compression or expansion times, so that the total cycle time can be approximated by $\tau_{\text {cycle }} \simeq$ $\tau_{1}+\tau_{3}=2 \tau$ for equal stroke duration. This assumption does not affect the generality of our results.

During the first and third strokes (compression and expansion), the quantum oscillator is isolated and only work is performed by changing the frequency in time. The mean work of the unitary dynamics can be evaluated by using the exact solution of the Schrödinger equation for the parametric oscillator for any given frequency modulation [49,50]. The mean work under scrutiny is then given by [8]

$$
\begin{aligned}
& \left\langle W_{1}\right\rangle=\frac{\hbar \omega_{2}}{2}\left(Q_{1}^{*}-\frac{\omega_{1}}{\omega_{2}}\right) \operatorname{coth}\left(\frac{\beta_{1} \hbar \omega_{1}}{2}\right), \\
& \left\langle W_{3}\right\rangle=\frac{\hbar \omega_{1}}{2}\left(Q_{3}^{*}-\frac{\omega_{2}}{\omega_{1}}\right) \operatorname{coth}\left(\frac{\beta_{2} \hbar \omega_{2}}{2}\right) .
\end{aligned}
$$

We have introduced the dimensionless quantities $Q_{1,3}^{*}$ that, by depending on the speed of the frequency driving [51], embodies a parameter of adiabaticity of the dynamics. In general, we have $Q_{1,3}^{*} \geqslant 1$, with the equality being satisfied for a quasistatic frequency modulation. Its expression is not crucial for the present analysis and can be found in Refs. [49,50], to which we refer for details.

During the thermalization steps (isochoric processes), heat is exchanged with the reservoirs. Such contributions can be quantified by calculating the corresponding variation of energy of the oscillator, which gives us

$$
\begin{aligned}
& \left\langle Q_{2}\right\rangle=\frac{\hbar \omega_{2}}{2}\left[\operatorname{coth}\left(\frac{\beta_{2} \hbar \omega_{2}}{2}\right)-Q_{1}^{*} \operatorname{coth}\left(\frac{\beta_{1} \hbar \omega_{1}}{2}\right)\right], \\
& \left\langle Q_{4}\right\rangle=\frac{\hbar \omega_{1}}{2}\left[\operatorname{coth}\left(\frac{\beta_{1} \hbar \omega_{1}}{2}\right)-Q_{3}^{*} \operatorname{coth}\left(\frac{\beta_{2} \hbar \omega_{2}}{2}\right)\right] .
\end{aligned}
$$

In order to operate as a refrigerator, the system should absorb heat from the cold reservoir, so that $\left\langle Q_{4}\right\rangle \geqslant 0$, and release it into the hot reservoir, which entails $\left\langle Q_{2}\right\rangle \leqslant 0$. According to Eq. (3), the condition for cooling is therefore that $\omega_{2} / \omega_{1}>\beta_{1} / \beta_{2}$.

The coefficient of performance $\epsilon$ of the quantum Otto refrigerator is given by the ratio of the heat removed from the cold reservoir to the total amount of work performed per cycle, $\epsilon=\left\langle Q_{4}\right\rangle /\left(\left\langle W_{1}\right\rangle+\left\langle W_{3}\right\rangle\right)$. It explicitly reads [8]

$$
\epsilon=\frac{\omega_{1}\left[\mathrm{c}\left(x_{1}\right)-Q_{3}^{*} \mathrm{c}\left(x_{2}\right)\right]}{\left(\omega_{2} Q_{1}^{*}-\omega_{1}\right) \mathrm{c}\left(x_{1}\right)-\left(\omega_{2}-\omega_{1} Q_{3}^{*}\right) \mathrm{c}\left(x_{2}\right)},
$$

where we have defined $x_{j}=\beta_{j} \hbar \omega_{j} / 2(j=1,2)$ and the function $\mathrm{c}\left(x_{1,2}\right)=\operatorname{coth}\left(x_{1,2}\right)$. For slow (adiabatic) driving processes, $Q_{1,2}^{*}=1$, the coefficient of performance of the 
refrigerator becomes [8]

$$
\epsilon_{\mathrm{AD}}=\frac{\omega_{1}}{\omega_{2}-\omega_{1}}
$$

which is positive provided that $\omega_{2}>\omega_{1}$.

An upper bound to the coefficient of performance in Eq. (4) follows from the second law of thermodynamics, which states that the total entropy production of a cyclic thermal device is non-negative [1]. Employing the quantum relative entropy of two density operators, $S\left(\rho_{1} \| \rho_{2}\right)=\operatorname{tr}\left\{\rho_{1} \ln \rho_{1}-\rho_{1} \ln \rho_{2}\right\} \geqslant$ 0 , the total entropy production for one complete cycle can be written as

$$
\begin{aligned}
\Delta S_{\mathrm{tot}} & =S\left(\rho_{A} \| \rho_{B}\right)+S\left(\rho_{B} \| \rho_{C}\right)+S\left(\rho_{C} \| \rho_{D}\right)+S\left(\rho_{D} \| \rho_{A}\right) \\
& =-\beta_{2}\left\langle Q_{2}\right\rangle-\beta_{1}\left\langle Q_{4}\right\rangle \geqslant 0,
\end{aligned}
$$

where we have assumed that the quantum relative entropy during the adiabatic processes $A B$ and $C D$ is negligible, as the von Neumann entropy is constant. Moreover, the quantum relative entropy of the isochoric processes $B C$ and $D A$ corresponds to the entropy production associated with the heating and cooling steps. From Eq. (4), the total entropy production is then $[42,47]$

$$
\Delta S_{\text {tot }}=x_{2}\left[Q_{1}^{*} \mathrm{c}\left(x_{1}\right)-\mathrm{c}\left(x_{2}\right)\right]-x_{1}\left[\mathrm{c}\left(x_{1}\right)-Q_{3}^{*} \mathrm{c}\left(x_{2}\right)\right] \geqslant 0 .
$$

Equality to zero is reached for the Carnot cycle scenario for which $\beta_{2} / \beta_{1}=\omega_{1} / \omega_{2}$ and $Q_{1,3}^{*}=1$. Based on the first law of thermodynamics, we have in addition

$$
-\left(\left\langle W_{1}\right\rangle+\left\langle W_{3}\right\rangle\right)=\left\langle Q_{2}\right\rangle+\left\langle Q_{4}\right\rangle .
$$

Combining Eqs. (6) and (8), we obtain the following upper bound on the refrigerator performance:

$$
\frac{\left\langle W_{1}\right\rangle+\left\langle W_{3}\right\rangle}{\left\langle Q_{4}\right\rangle} \leqslant \frac{\beta_{2}}{\beta_{1}-\beta_{2}}=\frac{T_{1}}{T_{2}-T_{1}}=\epsilon_{\mathrm{C}} .
$$

The above equation shows that the coefficient of performance of the quantum refrigerator is always bounded by the Carnot coefficient of performance.

\section{DRIVING A QUANTUM REFRIGERATOR WITH SHORTCUTS TO ADIABATICITY}

Let us now consider the situation when the compression and expansion strokes of the Otto refrigerator cycle is sped up by addition of a counterdiabatic driving control field $H_{\mathrm{STA}}^{\mathrm{CD}}(t)$ to the original harmonic oscillator Hamiltonian $H_{\mathrm{O}}(t)$. Scope of this term is to suppress the nonadiabatic transitions induced by the finite-time evolution of the oscillator and, as a consequence, quench the entropy production all the way down to the value taken in the adiabatic manifold of the initial system Hamiltonian. The resulting effective Hamiltonian reads $[11,12]$

$$
\begin{aligned}
H_{\mathrm{CD}}(t) & =H_{\mathrm{O}}(t)+H_{\mathrm{STA}}^{\mathrm{CD}}(t) \\
& =H_{\mathrm{O}}(t)+i \hbar \sum_{n}\left(\left|\partial_{t} n\right\rangle\left\langle n\left|-\left\langle n \mid \partial_{t} n\right\rangle\right| n\right\rangle\langle n|\right),
\end{aligned}
$$

where $|n\rangle \equiv|n(t)\rangle$ denotes the $n$th eigenstate of the original Hamiltonian $H_{\mathrm{O}}(t)$. For a harmonic working medium, the counterdiabatic term $H_{\mathrm{STA}}^{\mathrm{CD}}(t)$ is $[10,13]$

$$
H_{\mathrm{STA}}^{\mathrm{CD}}(t)=-\frac{\dot{\omega}_{t}}{4 \omega_{t}}(x p+p x) .
$$

Although this additional control removes the requirement of slow driving, the (nonlocal) counterdiabatic potential, which induces squeezing of the oscillator, makes its experimental application/implementation a challenging task. As a result, in order to circumvent this difficulty, it is natural to construct a unitarily equivalent Hamiltonian with a local potential. This is achieved by applying the operator $U_{x}=\exp \left(i m \dot{\omega}_{t} x^{2} / 4 \hbar \omega\right)$, which cancels the squeezing term and gives the new effective local counterdiabatic (LCD) Hamiltonian [15]

$$
H_{\mathrm{LCD}}(t)=U_{x}^{\dagger}\left(H_{\mathrm{CD}}(t)-i \hbar \dot{U}_{x} U_{x}^{\dagger}\right) U_{x}=\frac{p^{2}}{2 m}+\frac{m \Omega_{t}^{2} x^{2}}{2},
$$

where the modified time-dependent frequency is $\Omega^{2}(t)=$ $\omega_{t}^{2}-3 \dot{\omega}_{t}^{2} / 4 \omega_{t}^{2}+\ddot{\omega}_{t} / 2 \omega_{t}$. By requesting that the initial and final state of the working medium ensuing from $H_{\mathrm{LCD}}(t)$ equal that from the original Hamiltonian $H_{\mathrm{O}}(t)$, one gets the boundary conditions

$$
\begin{array}{lll}
\omega_{0}=\omega_{i}, & \dot{\omega}_{0}=0, & \ddot{\omega}_{0}=0, \\
\omega_{\tau}=\omega_{f}, & \dot{\omega}_{\tau}=0, & \ddot{\omega}_{\tau}=0,
\end{array}
$$

where $\omega_{i, f}=\omega_{1,2}$ correspond to the initial and final frequency of the compression/expansion strokes. A suitable ansatz is $[15,16]$

$\omega_{t}=\omega_{i}+10\left(\omega_{f}-\omega_{i}\right) s^{3}-15\left(\omega_{f}-\omega_{i}\right) s^{4}+6\left(\omega_{f}-\omega_{i}\right) s^{5}$

with $s=t / \tau$. In order to ensure that the trap is not inverted, one must also guarantee that $\Omega(t)^{2}>0$ is always fulfilled [27]. The mean value of the local counterdiabatic Hamiltonian $H_{\mathrm{LCD}}(t)$ may be calculated explicitly for an initial thermal state and reads [30]

$$
\begin{aligned}
\left\langle H_{\mathrm{LCD}}(t)\right\rangle & =\frac{\hbar \omega_{t}}{2}\left(1-\frac{\dot{\omega}_{t}^{2}}{4 \omega_{t}^{4}}+\frac{\ddot{\omega}_{t}}{4 \omega_{t}^{3}}\right) \operatorname{coth}\left(\frac{\beta \hbar \omega_{i}}{2}\right), \\
& =\frac{\omega_{t}}{\omega_{0}} Q_{\mathrm{LCD}}^{*}\langle H(0)\rangle,
\end{aligned}
$$

where we have introduced the LCD parameter

$$
Q_{\mathrm{LCD}}^{*}(t)=1-\frac{\dot{\omega}_{t}^{2}}{4 \omega_{t}^{4}}+\frac{\ddot{\omega}_{t}}{4 \omega_{t}^{3}} .
$$

The expectation value of the control field $H_{\mathrm{STA}}^{\mathrm{LCD}}(t)$ follows therefore as

$$
\left\langle H_{\mathrm{STA}}^{\mathrm{LCD}}(t)\right\rangle=\frac{\hbar \omega_{t}}{2}\left(-\frac{\dot{\omega}_{t}^{2}}{4 \omega_{t}^{4}}+\frac{\ddot{\omega}_{t}}{4 \omega_{t}^{3}}\right) \operatorname{coth}\left(\frac{\beta \hbar \omega_{i}}{2}\right),
$$

where we have used $\left\langle H_{\mathrm{O}}(t)\right\rangle=\hbar \omega_{t} \operatorname{coth}\left(\beta \hbar \omega_{i} / 2\right) / 2 \quad$ [50]. Based on the boundary conditions in Eq. (13), we have $\left\langle H_{\mathrm{STA}}^{\mathrm{LCD}}(\bar{t})\right\rangle=0$ for $\bar{t}=0$ and $\tau$, while the time-averaged value is non-null. We also remark that the local counterdiabatic control has been implemented in various experimental platforms [19,21], specifically in Paul traps [21], which are a potential candidate for building quantum thermal devices [52]. 


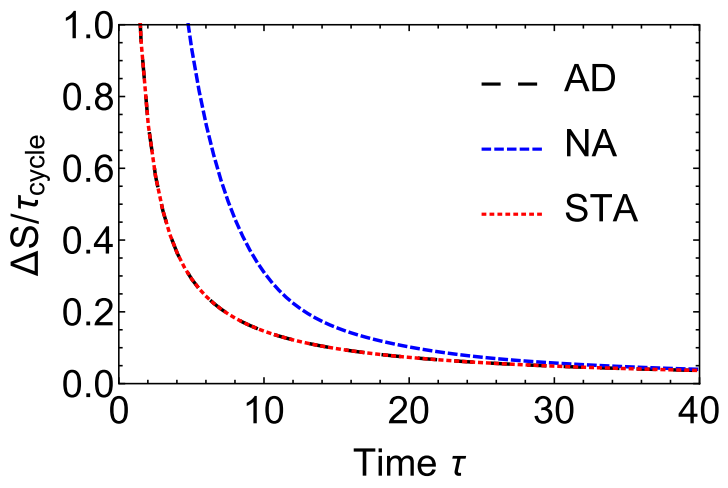

FIG. 2. Entropy production rate $\Delta S_{\text {tot }} / \tau_{\text {cycle }}$ of the quantum Otto refrigerator plotted against the driving time $\tau$. The blue (small dashed) line shows the nonadiabatic expression Eq. (7) in the absence of STA driving, while the red (dotted) line represents the corresponding result including STA techniques. Local counterdiabatic driving is seen to greatly reduce the irreversible entropy production rate to the adiabatic value (black large dashed). Parameters are $\hbar=1, \omega_{1}=$ $0.1, \omega_{2}=0.5, \beta_{1}=1$, and $\beta_{2}=0.75$.

Figure 2 shows the rate of entropy production $\Delta S_{\text {tot }} / \tau_{\text {cycle }}$ as a function of the time $\tau$ for adiabatic and nonadiabatic driving. We see that for short cycle time, the entropy production of nonadiabatic transition processes dramatically increases (blue dashed), thus leading to lower performance of the thermal machine. On the other hand, the application of STA methods is effective in suppressing such over-shooting of irreversible entropy (red dotted) to the adiabatic value (black large dashed).

\section{PERFORMANCE OF A SUPERADIABATIC QUANTUM REFRIGERATOR}

We now study three important quantities characterizing the performance of a refrigerator, namely its coefficient of performance $\varepsilon$, its cooling rate $J_{\text {STA }}^{c}$ and its figure of merit $\chi$. Taking into account the energetic cost of the STA driving, we define the coefficient of performance of the superadiabatic quantum Otto refrigerator as the ratio of the heat removed from the cold reservoir to the total amount of energy added per cycle

$$
\epsilon_{\mathrm{STA}}=\frac{\left\langle Q_{4}\right\rangle}{\sum_{i=1,3}\left(\left\langle W_{i}\right\rangle_{\mathrm{STA}}+\left\langle H_{\mathrm{STA}}^{i}\right\rangle_{\tau}\right)},
$$

where $\left\langle H_{\mathrm{STA}}^{i}\right\rangle_{\tau}=(1 / \tau) \int_{0}^{\tau} d t\left\langle H_{\mathrm{STA}}^{i}(t)\right\rangle(i=1,3)$, is the time average of the mean value of the local potential for the compression/expansion strokes and quantifies the energetic cost of the transitionless driving. When the energetic cost of the STA protocol is ignored [which corresponds to setting $\left\langle H_{\mathrm{STA}}^{i}\right\rangle_{\tau}=0$ in Eq. (18)], the coefficient of performance reduces to the adiabatic expression $\epsilon_{\mathrm{AD}}$ given by Eq. (5) $[8,32,40,45]$.

Figure 3 shows the coefficient of performance of the superadiabatic quantum refrigerator $\epsilon_{\mathrm{STA}}$ (red dotted) as a function of time $\tau$, together with the adiabatic $\epsilon_{\mathrm{AD}}$ (black large dashed) and nonadiabatic $\epsilon_{\mathrm{NA}}=\left\langle Q_{4}\right\rangle /\left(\left\langle W_{1}\right\rangle+\left\langle W_{3}\right\rangle\right)$ (blue small dashed) counterparts. We observe that the superadiabatic driving significantly enhances the performance of the quantum Otto refrigerator, $\epsilon_{\mathrm{NA}} \leqslant \epsilon_{\mathrm{STA}} \leqslant \epsilon_{\mathrm{AD}}$, for all driving

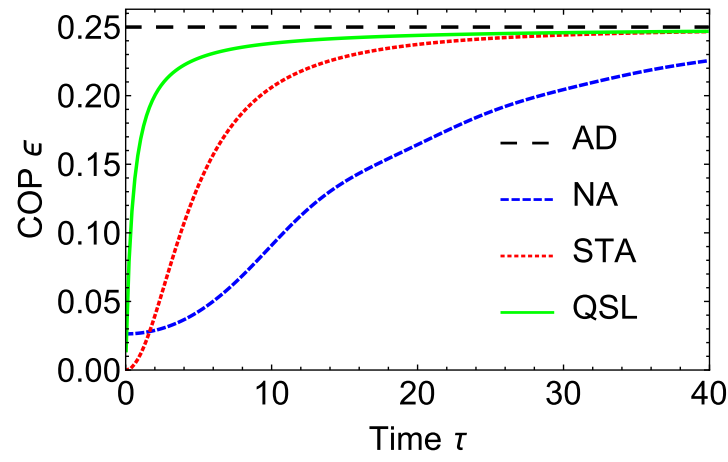

FIG. 3. Coefficient of performance of the quantum Otto refrigerator as a function of time $\tau$. The blue (small dashed) line shows the exact nonadiabatic case (NA), Eq. (4), while the red (dotted) line and the green (solid) lines respectively display the STA results, Eq. (18) and the quantum speed limit (QSL) bound, Eq. (21). The black (large dashed) line corresponds to the adiabatic case, Eq. (5). Same parameters as in Fig. 2.

times larger than $\tau \approx 2.0$, even though the energetic cost of the STA is explicitly included. We additionally note that the superadiabatic coefficient of performance $\epsilon_{\mathrm{STA}}$ is remarkably close to the adiabatic value $\epsilon_{\mathrm{AD}}$ for $\tau \geqslant 25$, indicating that the energetic STA cost is relatively small for larger times. Yet, the nonadiabatic coefficient of performance $\epsilon_{\mathrm{NA}}$ is already greatly reduced compared to the adiabatic value in this regime. The STA techniques thus appear here to be highly effective at suppressing nonadiabatic transitions at a little energetic cost.

On the other hand, the cooling power of the superadiabatic refrigerator is given by the ratio of heat flowing from the cold reservoir into the system to the cycle time

$$
J_{\text {STA }}^{c}=\frac{\left\langle Q_{4}\right\rangle_{\text {STA }}}{\tau_{\text {cycle }}} .
$$

An infinitely long cycle time, which would allow us to achieve the maximum coefficient of performance, would thus also give zero cooling power. In this regard, the main advantage of the STA approach is to realize the same amount of heat output as in the adiabatic case, but in a shorter cycle time. Hence, the STA strategy ensures that $J_{\mathrm{STA}}^{c}$ (red dotted) is always greater than the nonadiabatic cooling rate $J_{\mathrm{NA}}^{c}=\left\langle Q_{4}\right\rangle_{\mathrm{NA}} / \tau_{\text {cycle }}$ (blue dashed) for fast cycles, as shown in Fig. 4(a). However, there still exists a trade off between cooling power and coefficient of performance of STA refrigerator for fast cycles.

Following Feldmann and Kosloff [53], such trade off can be illustrated as in Fig. 4(b), where the dependence of $1 / \epsilon$ on the inverse cooling power $1 / J^{c}$ is illustrated for both the STA driving and the nonadiabatic protocol. The former simultaneously enhances both the coefficient of performance and the cooling power, thereby clearly demonstrating the benefits of the STA quantum Otto refrigerator over the conventional ones.

We finally consider the figure of merit $\chi=\epsilon\left\langle Q_{4}\right\rangle / \tau_{\text {cycle }}=$ $\epsilon J^{c}$ defined as the product of the coefficient of performance $\epsilon$ and the cooling power of the refrigerator $[6,8,42,43]$. The corresponding expression for a heat engine, $\chi_{\text {engine }}=$ $\eta\left\langle Q_{2}\right\rangle / \tau_{\text {cycle }}=-\langle W\rangle / \tau_{\text {cycle }}$, is equal to its power output $\left\langle Q_{2}\right\rangle$ being in this case the heat absorbed from the hot reservoir. In optimization problems, the maximum figure of merit (and 

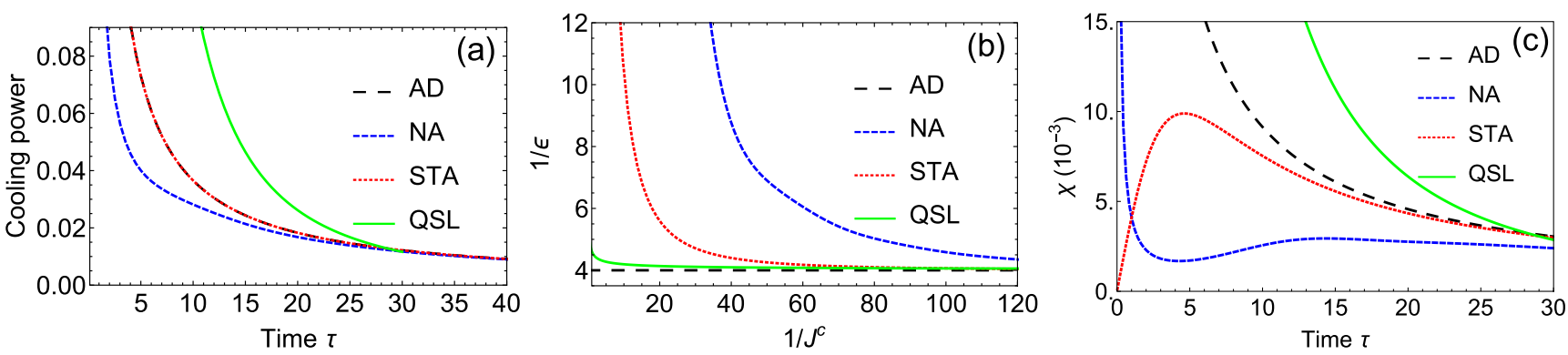

FIG. 4. (a) Cooling power of the quantum Otto refrigerator as a function of time $\tau$. The blue (dashed) line shows the exact nonadiabatic case, while the red (dotted) line and the green (solid) lines respectively represent the STA-based result in Eq. (19) and the quantum speed limit bound in Eq. (22). The black (large dashed) line is the adiabatic case, Eq. (4). (b) Inverse coefficient of performance as a function of the cooling power for the time duration $\tau=0.5-45$, for the same cases as in (a). (c) Figure of merit $\chi$ [see Eq. (23)] as a function of the driving time $\tau$ for the same cases as above. Same parameters as in Fig. 2.

not the maximum cooling power) condition for refrigerators is often used in analogy to the maximum power criterion for heat engines $[6,8,42,43]$. Figure 4 (c) presents the corresponding values as a function of $\tau$ for the case of adiabatic, nonadiabatic and STA strategies. Similar to the cooling power (19), a clear hierarchy emerges as $\chi_{\mathrm{NA}} \leqslant \chi_{\mathrm{STA}} \leqslant \chi_{\mathrm{AD}}$, except for short driving times, with the equality holding in the long-time limit. Compared to the nonadiabatic case, the STA approach increases the area under the curve, which determines the overall performance of the device.

\section{PERFORMANCE BOUNDS BY QUANTUM SPEED LIMIT}

The maximum performance of a classical thermal machine (refrigerator/engine) is limited by the second law of thermodynamics [2]. However, quantum mechanics imposes restrictions on the time of evolution of quantum processes. Understanding such restrictions is important for the successful implementation of STA techniques [30]. We next derive general upper bounds for both the STA-based coefficient of performance and the cooling rate of the quantum Otto refrigerator using the concept of quantum speed limits, which can be regarded as an extension of the energy-time uncertainty relation [38,54-57].

For unitary driven dynamics, a Margolus-Levitin-type bound [57] on the evolution time given by [58]

$$
\tau \geqslant \tau_{\mathrm{QSL}}=\frac{\hbar \mathcal{L}\left(\rho_{i}, \rho_{f}\right)}{\left\langle H_{\mathrm{STA}}\right\rangle_{\tau}},
$$

is appropriate. Here $\mathcal{L}\left(\rho_{i}, \rho_{f}\right)=\arccos \sqrt{F\left(\rho_{f}, \rho_{i}\right)}$ denotes the Bures angle between initial and final density operators of the system, with $F\left(\rho_{f}, \rho_{i}\right)$ the fidelity between the two, and $\left\langle H_{\mathrm{STA}}\right\rangle_{\tau}$ the time-averaged superadiabatic energy. Equation (20) becomes a proper bound for compression and expansion phases, when the refrigerator dynamics is dominated by the STA driving for small $\tau$.

From Eqs. (18) and (20), an upper bound on the STA-based coefficient of performance of the quantum Otto refrigerator is obtained as

$$
\epsilon_{\mathrm{STA}} \leqslant \epsilon_{\mathrm{STA}}^{\mathrm{QSL}}=\frac{\left\langle Q_{4}\right\rangle_{\mathrm{AD}}}{\left\langle W_{1}\right\rangle_{\mathrm{AD}}+\left\langle W_{3}\right\rangle_{\mathrm{AD}}+\hbar\left(\mathcal{L}_{1}+\mathcal{L}_{3}\right) / \tau},
$$

where $\mathcal{L}_{i}(i=1,3)$ are the respective Bures angles for compression/expansion steps. Likewise, an upper bound on the
STA-based cooling power [Eq. (19)] reads

$$
J_{\mathrm{STA}}^{c} \leqslant J_{\mathrm{STA}}^{c \mathrm{QSL}}=-\frac{\left\langle Q_{4}\right\rangle_{\mathrm{AD}}}{\tau_{\mathrm{QSL}}^{1}+\tau_{\mathrm{QSL}}^{3}},
$$

where $\tau_{\mathrm{OSL}}^{i}(i=1,3)$ are the respective speed-limit bounds in Eq. (20) for the compression/expansion phases. In addition, an upper bound for the figure of merit $\chi$ follows as

$$
\chi_{\mathrm{STA}} \leqslant \chi_{\mathrm{STA}}^{\mathrm{QSL}}=\epsilon_{\mathrm{STA}}^{\mathrm{QSL}} J_{\mathrm{STA}}^{c \mathrm{QSL}} .
$$

The above upper bounds are displayed in Figs. 3, 4(a), 4(b), and 4(c) (green solid). We observe that the quantum bound on the coefficient of performance [Figs. 3 and 4(b)] is much tighter than the adiabatic bound (black large dashed) imposed by the second law of thermodynamics (discussed in Sec. II). They are hence more useful for applications. We emphasize that these results are general and do not depend on the choice of the refrigerator cycle or on the STA driving protocol.

\section{CONCLUSIONS}

We have studied the performance of a quantum Otto refrigerator with a working medium consisting of a timedependent harmonic oscillator, exploiting STA mechanisms. We have explicitly analyzed the coefficient of performance, the cooling power, as well as the related figure of merit, for the case of local counterdiabatic driving. We have found that the STA quantum refrigerator outperforms its conventional nonadiabatic counterpart, except for short cycle durations, by strongly minimizing the nonequilibrium entropy production, even when the energetic cost of the STA driving is included. We have further derived generic upper bounds on the coefficient of performance of the Otto refrigerator by using the concept of quantum speed limits. Such bounds are tighter than those based on the second law and therefore more useful. The possibility to achieve simultaneous enhancements of coefficient of performance and cooling power should be of advantage for the future design of micro- and nanodevices operating in the quantum regime.

Note added in proof. The application of counterdiabatic driving techniques to speed up a quantum Otto refrigerator based on a superconducting qubit was recently studied in Ref. [59]. 


\section{ACKNOWLEDGMENTS}

We acknowledge support from the Royal Commission for the Exhibition of 1851, the EU Collaborative project TEQ (Grant Agreement No. 766900), the DfE-SFI Investigator Programme (Grant No. 15/IA/2864), COST Action
CA15220, the Royal Society Newton Fellowship (Grant No. NF160966), the Royal Society Wolfson Research Fellowship (RSWFIR3\183013), the Leverhulme Trust Research Project Grant (Grant No. RGP-2018-266) and the German Science Foundation (DFG) (Contract No. FOR 2724). We thank Nicolò Piccione for helpful discussions.
[1] H. Callen, Thermodynamics and an Introduction to Thermostatistics (Wiley, New York, 1985).

[2] Y. A. Cengel and M. A. Boles, Thermodynamics. An Engineering Approach (McGraw-Hill, New York, 2001).

[3] B. Andresen, Current trends in finite-time thermodynamics, Angew. Chem. Int. Ed. 50, 2690 (2011).

[4] Y. Apertet, H. Ouerdane, A. Michot, C. Goupil, and P. Lecoeur, On the efficiency at maximum cooling power, Europhys. Lett. 103, 40001 (2013).

[5] Z. Yan and J. Chen, A class of irreversible Carnot refrigeration cycles with a general heat transfer law, J. Phys. D: Appl. Phys. 23, 136 (1990).

[6] S. Velasco, J. M. M. Roco, A. Medina, and A. Calvo Hernández, New Performance Bounds for a Finite-Time Carnot Refrigerator, Phys. Rev. Lett. 78, 3241 (1997).

[7] N. Sanchez-Salas and A. Calvo Hernández, Harmonic quantum heat devices: Optimum-performance regimes, Phys. Rev. E 70, 046134 (2004).

[8] O. Abah and E. Lutz, Optimal performance of a quantum Otto refrigerator, Europhys. Lett. 113, 60002 (2016).

[9] X. Chen and J. G. Muga, Transient energy excitation in shortcuts to adiabaticity for the time-dependent harmonic oscillator, Phys. Rev. A 82, 053403 (2010).

[10] E. Torrontegui et al., Chapter 2. Shortcuts to adiabaticity, Adv. At. Mol. Opt. Phys. 62, 117 (2013).

[11] M. Demirplak and S. A. Rice, Adiabatic population transfer with control fields, J. Phys. Chem. A 107, 9937 (2003).

[12] M. V. Berry, Transitionless quantum driving, J. Phys. A: Math. Theor. 42, 365303 (2009)

[13] X. Chen, A. Ruschhaupt, S. Schmidt, A. del Campo, D. GuéryOdelin, and J. G. Muga, Fast Optimal Frictionless Atom Cooling in Harmonic Traps: Shortcut to Adiabaticity, Phys. Rev. Lett. 104, 063002 (2010).

[14] S. Masuda and K. Nakamura, Fast-forward of adiabatic dynamics in quantum mechanics, Proc. R. Soc. A 466, 1135 (2010).

[15] A. del Campo, Shortcuts to Adiabaticity by Counterdiabatic Driving, Phys. Rev. Lett. 111, 100502 (2013).

[16] S. Deffner, C. Jarzynski, and A. del Campo, Classical and Quantum Shortcuts to Adiabaticity for Scale-Invariant Driving, Phys, Rev. X 4, 021013 (2014).

[17] S. Campbell, G. De Chiara, M. Paternostro, G. M. Palma, and R. Fazio, Shortcut to Adiabaticity in the Lipkin-Meshkov-Glick Model, Phys. Rev. Lett. 114, 177206 (2015).

[18] M. Okuyama and K. Takahashi, Quantum-classical correspondence of shortcuts to adiabaticity, J. Phys. Soc. Jpn. 86, 043002 (2017).

[19] J.-F. Schaff, X.-L. Song, P. Capuzzi, P. Vignolo, and G. Labeyrie, Shortcut to adiabaticity for an interacting BoseEinstein condensate, Europhys. Lett. 93, 23001 (2011).
[20] M. G. Bason, M. Viteau, N. Malossi, P. Huillery, E. Arimondo, D. Ciampini, R. Fazio, V. Giovannetti, R. Mannella, and O. Morsch, High-fidelity quantum driving, Nat. Phys. 8, 147 (2012).

[21] A. Walther, F. Ziesel, T. Ruster, S. T. Dawkins, K. Ott, M. Hettrich, K. Singer, F. Schmidt-Kaler, and U. Poschinger, Controlling Fast Transport of Cold Trapped Ions, Phys. Rev. Lett. 109, 080501 (2012).

[22] S. An, D. Lv, A. del Campo, and K. Kim, Shortcuts to adiabaticity by counterdiabatic driving in trapped-ion transport, Nat. Commum. 7, 12999 (2016).

[23] Y.-X. Du, Z.-T. Liang, Y.-C. Li, X.-X. Yue, Q.-X. Lv, W. Huang, X. Chen, H. Yan, and S.-L. Zhu, Experimental realization of stimulated Raman shortcut-to-adiabatic passage with cold atoms, Nat. Commun. 7, 12479 (2016).

[24] D. Stefanatos, Minimum-time transitions between thermal and fixed average energy states of the quantum parametric oscillator, SIAM J. Control Optim. 55, 1429 (2017).

[25] J. Deng, Q.-H. Wang, Z. Liu, P. Hänggi, and J. Gong, Boosting work characteristics and overall heat-engine performance via shortcuts to adiabaticity: Quantum and classical systems, Phys. Rev. E 88, 062122 (2013).

[26] Z. C. Tu, Stochastic heat engine with the consideration of inertial effects and shortcuts to adiabaticity, Phys. Rev. E 89, 052148 (2014).

[27] A. del Campo, J. Goold, and M. Paternostro, More bang for your buck: Super-adiabatic quantum engines, Sci. Rep. 4, 6208 (2014).

[28] M. Beau, J. Jaramillo, and A. del Campo, Scaling-up quantum heat engines efficiently via shortcuts to adiabaticity, Entropy 18, 168 (2016).

[29] L. Chotorlishvili, M. Azimi, S. Stagraczynski, Z. Toklikishvili, M. Schüler, and J. Berakdar, Superadiabatic quantum heat engine with a multiferroic working medium, Phys. Rev. E 94, 032116 (2016)

[30] O. Abah and E. Lutz, Energy efficient quantum machines, Europhys. Lett. 118, 40005 (2017).

[31] O. Abah and E. Lutz, Performance of shortcut-toadiabaticity quantum engines, Phys. Rev. E 98, 032121 (2018).

[32] Y. Rezek, P. Salamon, K. H. Hoffmann, and R. Kosloff, The quantum refrigerator: The quest for absolute zero, Europhys. Lett. 85, 30008 (2009).

[33] Y. Zheng, S. Campbell, G. De Chiara, and D. Poletti, Cost of counterdiabatic driving and work output, Phys. Rev. A 94, 042132 (2016).

[34] I. B. Coulamy, A. C. Santos, I. Hen, and M. S. Sarandy, Energetic cost of superadiabatic quantum computation, Front. ICT 3, 19 (2016). 
[35] J. Li, T. Fogarty, S. Campbell, X. Chen, and T. Busch, An efficient nonlinear Feshbach engine, New J. Phys. 20, 015005 (2018).

[36] B. Cakmak and Ö. E. Müstecaplioglu, Spin quantum heat engines with shortcuts to adiabaticity, Phys. Rev. E 99, 032108 (2019).

[37] A. Tobalina, I. Lizuain, and J. G. Muga, Vanishing efficiency of speeded-up quantum Otto engines, Europhys. Lett. 127, 20005 (2019).

[38] S. Deffner and S. Campbell, Quantum speed limits: From Heisenberg's uncertainty principle to optimal quantum control, J. Phys. A: Math. Theor. 50, 453001 (2017).

[39] B. Karimi and J. P. Pekola, Otto refrigerator based on a superconducting qubit: Classical and quantum performance, Phys. Rev. B 94, 184503 (2016).

[40] J. Birjukov, T. Jahnke, and G. Mahler, Quantumthermodynamic processes: A control theory for machine cycles, Eur. Phys. J. B 64, 105 (2008).

[41] R. Kosloff and T. Feldmann, Optimal performance of reciprocating demagnetization quantum refrigerators, Phys. Rev. E 82, 011134 (2010).

[42] A. E. Allahverdyan, K. Hovhannisyan, and G. Mahler, Optimal refrigerator, Phys. Rev. E 81, 051129 (2010).

[43] C. de Tomás, A. Calvo Hernández, and J. M. M. Roco, Optimal low symmetric dissipation Carnot engines and refrigerators, Phys. Rev. E 85, 010104 (2012).

[44] O. Abah, J. Rossnagel, G. Jabob, S. Deffner, F. Schmidt-Kaler, K. Singer, and E. Lutz, Single-Ion Heat Engine at Maximum Power, Phys. Rev. Lett. 109, 203006 (2012).

[45] Y. Yuan, R. Wang, J. He, Y. Ma, and J. Wang, Coefficient of performance under maximum $\chi$ criterion in a two-level atomic system as a refrigerator, Phys. Rev. E 90, 052151 (2014).
[46] B. Lin, and J. Chen, Optimal analysis on the performance of an irreversible harmonic quantum Brayton refrigeration cycle, Phys. Rev. E 68, 056117 (2003).

[47] Y. Rezek and R. Kosloff, The quantum harmonic Otto cycle, Entropy 19, 136 (2016).

[48] O. Abah and M. Paternostro, Shortcut-to-adiabaticity Otto engine: A twist to finite-time thermodynamics, Phys. Rev. E 99, 022110 (2019).

[49] S. Deffner and E. Lutz, Nonequilibrium work distribution of a quantum harmonic oscillator, Phys. Rev. E 77, 021128 (2008).

[50] S. Deffner, O. Abah, and E. Lutz, Quantum work statistics of linear and nonlinear parametric oscillators, Chem. Phys. 375, 200 (2010).

[51] K. Husimi, Miscellanea in elementary quantum mechanics II, Prog. Theor. Phys. 9, 381 (1953).

[52] J. Rossnagel, S. T. Dawkins, K. N. Tolazzi, O. Abah, E. Lutz, F. Schmidt-Kaler, and K. Singer, A single-atom heat engine, Science 352, 325 (2016).

[53] T. Feldmann and R. Kosloff, Short time cycles of purely quantum refrigerators, Phys. Rev. E 85, 051114 (2012).

[54] J. Anandan and Y. Aharonov, Geometry of Quantum Evolution, Phys. Rev. Lett. 65, 1697 (1990).

[55] L. Vaidman, Minimum time for the evolution to an orthogonal quantum state, Am. J. Phys. 60, 182 (1992).

[56] J. Uffink, The rate of evolution of a quantum state, Am. J. Phys. 61, 935 (1993).

[57] N. Margolus and L. B. Levitin, The maximum speed of dynamical evolution, Physica D 120, 188 (1998).

[58] S. Deffner and E. Lutz, Energy-time uncertainty relation for driven quantum systems, J. Phys. A 46, 335302 (2013).

[59] K. Funo, N. Lambert, B. Karimi, J. P. Pekola, Y. Masuyama, and F. Nori, Speeding up a quantum refrigerator via counterdiabatic driving, Phys. Rev. B 100, 035407 (2019). 